Poznańskie Studia Teologiczne 29(2015), s. 7-15. doi: $10.14746 /$ pst.2015.29.1

S. Em. Angelo Card. Amato ${ }^{1}$

Congregazione delle Cause dei Santi, Città del Vaticano

\title{
La Dichiarazione «Dominus Iesus» a quindici anni dalla pubblicazione (2000-2015)
}

\section{Reazioni alla Dominus Iesus}

Pubblicata in piena celebrazione del Grande Giubileo dell'Anno Duemila (6 agosto 2000), la Dichiarazione Dominus Iesus, della Congregazione per la Dottrina della Fede, ${ }^{2}$ sottoscritta dall'allora Prefetto, Cardinale Joseph Ratzinger, e controfirmata, dall'allora Segretario del Dicastero, arcivescovo Tarcisio Bertone, suscitò sin dalla sua apparizione un accesissimo dibattito. Furono molte le adesioni, ma non mancarono - e furono vivaci a immediate - le reazioni e le critiche sul linguaggio troppo assertivo, sul contenuto poco dialogico, sulla inopportunità della sua pubblicazione.

Tali reazioni mostrano un triplice paradosso. Anzitutto, esse provenivano dall'ambiente teologico e "missionario" cattolico, il cui atteggiamento, invece, avrebbe dovuto essere, oltre che di adesione al magistero, soprattutto di riflessione sui motivi che avevano spinto la Congregazione a una tale puntualizzazione dottrinale.

Il secondo paradosso derivava dal fatto che il documento non proponeva una nuova dottrina, ma raccoglieva e organizzava alcune indicazioni magisteriali del Vaticano II e della Redemptoris Missio di Papa Giovanni Paolo II circa l'unicità e l'universalità salvifica di Gesù Cristo e della Chiesa.

Cosa conteneva la Dominus Iesus? In concreto, rispondeva ad alcune diffuse teorie di tipo relativistico della teologia delle religioni, che intendevano giustifi-

\footnotetext{
${ }^{1}$ Angelo kardynał Amato, salezjanin, prefekt Kongregacji ds. Beatyfikacji i Kanonizacji, w latach 2002-2008 pełnił funkcję sekretarza Kongregacji Nauki Wiary, Autor wielu dzieł z zakresu teologii dogmatycznej, szczególnie chrystologii, mariologii i eklezjologii.

${ }^{2}$ Congregazione per la Dottrina della Fede, Dichiarazione "Dominus Iesus", circa l'unicità e l'universalità salvifica di Gesù Cristo e della Chiesa, Libreria Editrice Vaticana, Città del Vaticano 2000. Nel testo la citeremo con i numeri tra parentesi. Cf. anche Congregazione per la Dottrina della Fede, Dichiarazione "Dominus Iesus". Documenti e studi, Libreria Editrice Vaticana, Città del Vaticano 2002; Pontificia Academia Theologica, "Gesù Cristo, via, verità e vita" (Gv 14,6). Per una rilettura della “Dominus Iesus”, PATH 1 (2002) n. 2, p. 141-370.
} 
care il pluralismo religioso non solo de facto ma anche de iure. Ecco un elenco di tali teorie fatto dalla Dichiarazione:

Si ritengono superate verità come, ad esempio, il carattere definitivo e completo della rivelazione di Gesù Cristo, la natura della fede cristiana rispetto alla credenza nelle altre religioni, il carattere ispirato dei libri della Sacra Scrittura, l'unità personale tra il Verbo eterno e Gesù di Nazareth, l'unità dell'economia del Verbo incarnato e dello Spirito Santo, l'unicità e l'universalità savifica del mistero di Gesù Cristo, la mediazione salvifica universale della Chiesa, l'inseparabilità, pur nella distinzione, tra il Regno di Dio, Regno di Cristo e la Chiesa, la sussistenza nella Chiesa cattolica dell'unica Chirsa di Cristo (n. 4).

Per contrastare queste affermazioni erronee, la Dichiarazione riaffermava la pienezza e la definitività della rivelazione di Gesù Cristo, contro le teorie della complementarietà di rivelazioni non cristiane (n. 6-8).

Ribadiva, in secondo luogo, l'unità e l'unicità dell'economia salvifica voluta da Dio Trinità, alla cui fonte e al cui centro c'è il mistero dell'incarnazione, rigettando quindi l'ipotesi di una economia dello Spirito Santo, con un raggio d'azione più ampio e una efficacia più universale di quella del Verbo incarnato (n. 9-12).

Riaffermava, poi, l'unicità e l'universalità del mistero salvifico di Gesù Cristo: «In nessun altro c'è salvezza; non vi è infatti altro nome dato agli uomini sotto il cielo nel quale dobbiamo essere salvati» (At 4,12) (n. 13-15).

In quarto luogo, ribadiva l'unicità della Chiesa di Cristo, che sussiste nella Chiesa Cattolica, governata dal Successore di Pietro e dai Vescovi in comunione con lui (n. 16-17). A tale riguardo si precisava che quelle Chiese non in perfetta comunione con la Chiesa Cattolica, restavano, purtuttavia, unite ad essa per mezzo di strettissimi vincoli, quali la successione apostolica e la valida Eucaristia, e quindi erano "vere Chiese particolari" (n. 17). Mentre quelle "comunità ecclesiali", che non avevano conservato l'episcopato valido e la genuina e integra sostanza del mistero eucaristico, "non sono Chiese in senso proprio" (n. 17).

La Dichiarazione, infine, riaffermava la necessità della Chiesa in ordine alla salvezza, dal momento che essa è sacramento universale di salvezza (n. 18-22).

Il terzo paradosso consisteva nel fatto che il documento non intendeva bloccare o chiudere la riflessione sulla teologia delle religioni, ma anzi suggeriva ulteriori piste di ricerca e di riflessione. Ad esempio, invitava i teologi «ad esplorare se e come anche figure ed elementi positivi di altre religioni rientrino nel piano divino di salvezza» (n. 14). A proposito, poi, della volontà salvifica universale di Dio, la Dichiarazione riconosceva che la teologia sta cercando di approfondire il tema del rapporti dei non cristiani con la grazia salvifica di Cristo e con la Chiesa (n. 21). 


\section{Una valutazione}

Cosa dire, oggi, a proposito della Dominus Iesus? A quindici anni di distanza si possono avanzare alcune considerazioni. La prima è che la Dichiarazione ha fatto chiarezza su alcune fondamentali verità cristologiche ed ecclesiologiche, sottraendole al "porto delle nebbie", e cioè alla negazione, alla confusione e all'equivoco dei teologi del pluralismo religioso.

Essa, inoltre, non solo non ha bloccato o condizionato negativamente i dialoghi ecumenici e interreligiosi, ma li ha rilanciati a partire da una precisa e riaffermata identità cattolica. Il miglior passaporto per entrare in territorio altrui è una leale carta d'identità, senza omissioni e senza falsificazioni. In tal modo i nostri interlocutori sono informati sui pilastri irrinunciabili della fede cristiana.

Una terza considerazione riguarda la pista di ricerca relativa alla salvezza dei non cristiani. La teologia pluralistica, che nega il dato biblico di Cristo salvatore universale, proponendo invece una pluralità di salvatori equivalenti, non corrisponde al piano divino trinitario. È solo nel mistero di Cristo che Dio Trinità ha predestinato, chiamato e giustificato l'intero genere umano.

\section{Una legittima preoccupazione: la salvezza dei non cristiani}

Ma c'è una preoccupazione, che ritengo legittima, proveniente dai teologi pluralisti e che - come abbiamo già accennato - la Dichiarazione considera degna di studio ulteriore. È la questione della salvezza dei non cristiani, della sorte, cioè, di quelle persone non battezzate, ma oneste, compassionevoli, di coscienza retta. Qual è il loro destino? Sono fuori o dentro l'influsso salvifico di Cristo? Insomma, come si compone l'universalità indiscussa del sacrificio redentore di Cristo con la moltitudine dei non cristiani, non pochi dei quali danno esempio di vita umana e religiosa esemplare?

Nell'esortazione postsinodale Ecclesia in Asia (1999), Giovanni Paolo II aveva offerto l'orientamento generale a queste domande, affermando: «Dal primo istante del tempo sino all'ultimo, Gesù è il solo Mediatore universale. Anche per quanti non professano esplicitamente la fede in lui quale Salvatore, la salvezza giunge da lui come grazia, mediante la comunicazione dello Spirito Santo».3

La questione, del resto, era stata affrontata anche dal Vaticano II. Il Decreto Ad Gentes, ad esempio, dopo aver messo in risalto l'obbligo della Chiesa di proclamare il mistero salvifico di Cristo, perché solo in lui c'è la salvezza per tutti (cf. At 4,12), dichiarava che «Deus, viis sibi notis, homines, Evangelium sine eorum culpa ignorantes, ad fidem adducere possit, sine qua impossibile est Ipsi placere». ${ }^{4}$ È un' affermazione sorprendente sulle vie segrete di Dio, che permette che giunga alla vera fede anche chi ignora, non per colpa sua, il Vangelo.

\footnotetext{
${ }^{3}$ Giovanni Paolo II, Esort. Postsin. Ecclesia in Asia, Città del Vaticano 1999, n. 14.

${ }^{4}$ Concilio Ecumenico Vaticano II, Dich. Ad gentes, data i miejsce wydania, n. 7a.
} 
Tale asserzione trova la sua esplicitazione nella Gaudium et spes che, dopo aver rilevato che anche nel cuore degli uomini di buona volontà lavora invisibilmente la grazia, continua: «Cristo, infatti, è morto per tutti e la vocazione ultima dell'uomo è effettivamente una sola, quella divina, perciò dobbiamo ritenere che lo Spirito Santo dia a tutti la possibilità di venire a contatto, nel modo che Dio conosce ("modo Deo cognito"), col mistero pasquale».5

Abbiamo, quindi, due indicazioni su un certo segreto salvifico di Dio. I testi conciliari parlano di un'azione divina, invisibile ma reale ed efficace, affinché anche i non cristiani possano raggiungere la fede e venire in contatto col mistero pasquale: per vie segrete ("viis sibi notis") e nel modo che solo Dio conosce ("modo Deo cognito"). ${ }^{6}$

\section{Le vie segrete di Dio}

Lo studio approfondito degli Acta Synodalia, alla ricerca delle motivazioni di queste affermazioni, hanno individuato almeno cinque vie mediante le quali Dio offre a tutti la possibilità di accedere a Cristo e alla Chiesa.

La prima è l'ordinazione alla Chiesa, sacramento universale di salvezza: «ad essa, in vari modi appartengono, oppure ad essa sono ordinati sia i fedeli cattolici, sia gli altri credenti in Cristo, sia, infine, tutti gli uomini che la grazia di Dio chiama alla salvezza» («sive denique omnes universaliter homines, gratia Dei ad salutem vocati»). ${ }^{7}$ La grazia redentrice di Cristo è infatti presente ed efficace nell'intera umanità, mediante il suo sacrificio eucaristico offerto per tutti.

L'obbedienza alla retta coscienza, che è la voce di Dio iscritta nel cuore umano, è una seconda possibilità di salvezza offerta a tutti gli esseri umani: «L'uomo ha in realtà una legge scritta da Dio nel suo cuore: obbedire ad essa è la dignità stessa dell'uomo, e secondo questa egli sarà giudicato». ${ }^{8}$

Una terza via segreta di salvezza è fare il bene ed evitare il male: «Nell'intimo della coscienza l'uomo scopre una legge che non è lui a darsi, ma alla quale invece deve obbedire e la cui voce, che lo chiama sempre ad amare e a fare il bene e a fuggire il male, quando occorre, chiaramente dice alle orecchie del cuore: Fa' questo, fuggi quest'altro». ${ }^{9}$

\footnotetext{
${ }^{5}$ Concilio Ecumenico Vaticano II, Cost. past. Gaudium et spes, data i miejsce wydania,n. 22e.

${ }^{6}$ Oltre ai testi conciliari, abbiamo le richieste dei Padri conciliari: cf., ad esempio, Acta Synodalia, Città del Vaticano 1977, IV/IV p. 414, 470. Per una visione completa di questa problematica, cf. una tesi di dottorato del salesiano Francis Fernandez, In Ways Known to God, Vendrame Institute Publications, Shillong 1996, pp. 328.

${ }^{7}$ Concilio Ecumenico Vaticano II, Cost. dogm. Lumen gentium, Bologna 1981, n. 13d.

${ }^{8}$ Concilio Ecumenico Vaticano II, Cost. past. Gaudium et spes, Bologna 1981, n. 16.

${ }^{9} \mathrm{Ib}$.
} 
Gli Acta Synodalia e i testi conciliari fanno emergere poi altre due vie segrete di salvezza, che sono: a de si on e alla ve rità, dal momento che la verità si impone da se stessa, vincendo con gentilezza e rispetto ogni resistenza contraria; e la coerenza tra fede e vita, per cui, ad esempio, il cristiano può perdersi, se non persevera nella verità e nella carità, mentre, viceversa, il non cristiano può salvarsi, se persevera nella verità e nella carità.

Queste indicazioni conciliari permettono di superare la falsa immagine di un cristianesimo autoritario e arrogante e di una Chiesa chiusa nei confronti di quelle tracce di verità e di bontà presenti fuori di essa. Il mistero redentore di Cristo ha valenza universale, e in modo misterioso, ma reale, raggiunge le menti e i cuori non solo dei battezzati, ma di tutti gli esseri umani. Se i mezzi di salvezza vengono offerti con abbondanza nella Chiesa, anche fuori di essa non mancano vie attraverso le quali Dio attrae le anime alla grazia e alla fede.

\section{La necessità della missio ad gentes}

Questo significa la cessazione dell'opera missionaria e dell'invito al battesimo e alla conversione, come gli autori pluralistici affermano? Il dialogo interreligioso sostituisce la missio ad gentes? La Chiesa deve rinunciare alla proclamazione del Vangelo e all'invito alla conversione e al battesimo?

Dando seguito alla Dominus Iesus, la Congregazione per la Dottrina della Fede pubblicò nel 2007 una Nota dottrinale su alcuni aspetti dell'evangelizzazione, in cui rimotiva l'urgenza e anzi la necessità oggi della missio ad gentes, che, nonostante duemila anni di cristianesimo, è ancora agli inizi. ${ }^{10} \mathrm{Il}$ dialogo interreligioso non può sostituire la proclamazione del Vangelo per alcune considerazioni di ordine antropologico.

\section{Considerazioni antropologiche}

Il cristiano non può occultare la propria identità di fede, così come l'interlocutore non cristiano non può nascondere le proprie credenze. La missio, da parte cristiana, è un invito rivolto al libero arbitrio dell'uomo e alla sua aspirazione al bene e al vero: «Nulla come la ricerca del bene e della verità mette in gioco la libertà umana, sollecitandola ad un'adesione tale da coinvolgere gli aspetti fondamentali della vita. Questo è in modo particolare il caso della verità salvifica, che non è soltanto oggetto del pensiero ma avvenimento che investe tutta la persona - intelligenza, volontà, sentimenti, attività e progetti - quando essa aderisce

\footnotetext{
${ }^{10}$ Congregazione per la Dottrina della Fede, Nota dottrinale su alcuni aspetti dell'Evangelizzazione, 3 dicembre 2007. Cf. i commenti in Congregazione per la Dottrina della Fede, Nota dottrinale su alcuni aspetti dell'evangelizzazione, Libreria Editrice Vaticana, Città del Vaticano 2009.
} 
a Cristo». ${ }^{11}$ La libertà non è indifferenza ma tensione al bene. In questa tensione verso il bene e il vero è già all'opera lo Spirito Santo, «che crea affinità ed avvicina i cuori alla verità, aiutando la conoscenza umana a maturare in sapienza e in abbandono fiducioso al vero». ${ }^{12}$

In secondo luogo, talvolta, si obietta sulla legittimità di proporre agli altri quanto si ritiene vero per sé stessi. Ciò viene considerato come un attentato all'altrui libertà di coscienza. In realtà, questa comprensione della libertà come svincolata dalla verità, è una delle espressione di quel relativismo gnoseologico che ammette solo opinioni senza alcun riferimento alla loro conformità alla realtà e alla verità. Si tratta di un atteggiamento di sfiducia nei confronti della verità: «Se l'uomo nega la sua fondamentale capacità della verità, se diviene scettico sulla sua facoltà di conoscere realmente ciò che è vero, egli finisce per perdere ciò che in modo unico può avvincere la sua intelligenza ed affascinare il suo cuore». ${ }^{13} \mathrm{Il}$ rispetto per la libertà religiosa altrui non può significare indifferenza verso la verità e il bene.

Inoltre, la ricerca della verità è compito specifico, non solo delle forze del singolo interlocutore, ma anche riconoscimento dell'esperienza altrui: «In particolare, la verità che è in grado di illuminare il senso della propria vita e di guidarla viene raggiunta anche mediante l»abbandono fiducioso a coloro che possono garantire la certezza e l'autenticità della verità stessa». ${ }^{14}$ Il cristiano che accoglie nella fede la rivelazione divina partecipa a questo dinamismo di ricerca della verità ma anche di annuncio e di comunicazione di questa verità salvifica agli altri. La sollecitazione dell'intelligenza e della libertà dell'interlocutore all'incontro con Cristo e con la sua parola di verità non costituisce una intrusione illecita, bensì una legittima offerta e un gratuito servizio al prossimo, sempre tenendo presente la massima conciliare che la verità «non si impone che in forza della stessa verità». ${ }^{15}$

Di conseguenza, la missio, e cioè la comunicazione delle verità religiose della fede cristiana, non è solo in sintonia con la natura dialogica di ogni essere umano, ma risponde a un'altra significativa realtà antropologica, quella di mostrare agli altri i propri beni e di renderli partecipi. Ora la verità salvifica spinge la libertà e la generosità dei fedeli a ridonare al prossimo quanto si è gratuitamente ricevuto. La volontà salvifica universale di Dio è un dato biblico innegabile e la grazia di Dio conosce vie misteriose per raggiungere i cuori dei non cristiani. Ma

\footnotetext{
${ }^{11}$ Congregazione per la Dottrina della Fede, Nota dottrinale su alcuni aspetti dell'evangelizzazione, n. 4.

${ }^{12} \mathrm{Ib}$.

${ }^{13} \mathrm{Ib}$.

${ }^{14}$ Ib. n. 5

${ }^{15}$ Concilio Vaticano II, Dich. Dignitatis humanae, Bologna 1981, n. 3.
} 
la Chiesa non può non tener conto del fatto che ad essi manca un grandissimo bene in questo mondo: conoscere il vero volto di Dio e l'amicizia con Gesù Cristo, il Diocon-noi. Infatti, "non vi è niente di più bello che essere raggiunti, sorpresi dal Vangelo, da Cristo. Non vi è niente di più bello che conoscere Lui e comunicare agli altri l'amicizia con Lui". ${ }^{16}$

La conoscenza della verità su Dio, sul destino dell'uomo e del cosmo, è un bene che sostiene l'esistenza di ogni essere umano. È come un esodo dall'oscurità dell'ignoranza alla luce della verità. La conoscenza della verità salvifica non umilia la libertà umana, ma la esalta estendendola verso il compimento delle sue aspirazioni alla felicità. Si tratta di un bene che la missio ad gentes vuole rendere partecipi tutti nella libertà e nel rispetto assoluto dell'altrui coscienza.

Il movente della missione evangelizzatrice della Chiesa è far conoscere l'amore di Cristo per la salvezza dell'umanità intera. E l'annuncio va fatto nella verità, nella libertà, nell'amicizia e nel rispetto delle ragioni e dei sentimenti altrui. Perciò «la Chiesa proibisce severamente di costringere o di indurre e attirare qualcuno con inopportuni raggiri ad abbracciare la fede, allo stesso modo che rivendica energicamente il diritto che nessuno con ingiuste vessazioni sia distolto dalla fede stessa"». ${ }^{17}$ L'interlocutore non cristiano non può intendere il dialogo come uno strumento per paralizzare o impedire l'annuncio cristiano.

\section{L'annuncio della verità nella carità}

In conclusione, gli ingredienti essenziali di ogni evangelizzazione sono i quattro principi della verità, della libertà, della carità e del rispetto dell'altrui coscienza. Per questo l'apostolo Paolo esortava gli Efesini a essere veritieri nella carità:

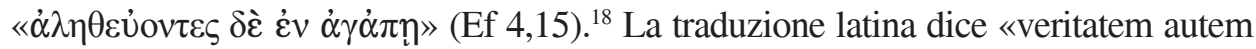
facientes in caritate» e quella italiana «vivendo secondo la verità nella carità». Si tratta in realtà di una «comunicazione veritiera», che è allo stesso tempo manifestazione concreta della verità nelle parole, nella vita e nelle opere. Con ciò san Paolo - commenta Heinrich Schlier - invita i fedeli a evitare «l'incertezza e la confusione di una fede malsicura di fronte alle idee brillanti e trascinatrici del tempo». ${ }^{19}$

L'annuncio della verità evangelica e la proclamazione del mistero salvifico di Cristo, fatto con sincerità e coraggio, devono essere sempre ancorati alla carità. È la carità la casa della verità e viceversa, dal momento che Dio è Carità e Verità.

\footnotetext{
${ }^{16}$ Congregazione per la Dottrina della Fede, Nota dottrinale su alcuni aspetti dell'evangelizzazione, n. 7.

${ }^{17}$ Ib. n. 8. La citazione si riferisce al Decreto Ad gentes, n. 13 del Concilio Vaticano II.

${ }^{18} \mathrm{Cf}$. R. Bultmann, $\dot{\alpha} \lambda \eta \theta \varepsilon \dot{\varepsilon} o v \tau \varepsilon \varsigma \delta \dot{\varepsilon} \dot{\varepsilon} v \dot{\alpha} \gamma \dot{\alpha} \pi \eta$, in Grande Lessico del Nuovo Testamento, Paideia, Brescia 1965, vol. I col. 674.

${ }^{19}$ H. Schlier, Lettera agli Efesini, Paideia, Brescia 1965, p. 248.
} 
In conclusione, la Dominus Iesus resta, oggi più che mai, un valido richiamo di chiarezza dottrinale e pastorale, come base della catechesi, della nuova evangelizzazione e della missio ad gentes. Occorre, però, una rieducazione teologica all'accoglienza del magistero come locus theologicus non secondario del fare teologia.

La recezione del magistero, soprattutto pontificio, è un tema di autentica formazione sacerdotale. «Sentire cum Ecclesia, amare Ecclesiam, criteria habere quae Ecclesia habet» è l'eredità dei Padri della Chiesa e dei grandi teologi di ogni tempo e soprattutto è l'atteggiamento dei Santi.

\section{Streszczenie}

Artykuł pt. Deklaracja „Dominus Iesus” po piętnastu latach od publikacji (2000-2015) jest analizą recepcji dokumentu Kongregacji Nauki Wiary w Kościele. Ponieważ deklaracja wzbudziła wiele komentarzy i krytycznych obserwacji na forach teologicznych całego świata, artykuł nie tylko je sygnalizuje, lecz również stanowi usystematyzowaną odpowiedź na pojawiające się zastrzeżenia i uwagi. Artykuł porusza między innymi kwestie natury soteriologicznej, dotyczące zbawienia niechrześcijan, zwraca uwagę na konieczność kontynuacji misji ad gentes, zachęca do kontynuacji dialogu międzyreligijnego.

\section{Keywords}

Congregation for the Doctrine of the Faith, Declaration Dominus Jesus, salvation, Christ, the Church

\section{Slowa kluczowe}

Kongregacja Nauki Wiary, Deklaracja Dominus Jesus, zbawienie, Chrystus, Kościół

\section{Bibliografia}

Acta synodalia S. Concilii oecumenici Vaticani II. Vol. 4/4: Congregationes generales CXLVI-CL. Libreria Editrice Vaticana, Città del Vaticano 1977.

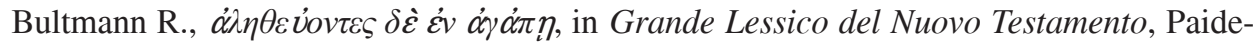
ia, Brescia 1965, vol. I.

Concilio Ecumenico Vaticano II, Costituzione dogmatica su la Chiesa Lumen gentium, in: Enchridion Vaticanum. Documenti ufficiali del Concilio Vaticano II (1962-1965), vol. I, EDB, Bologna 1981, pp. 120-263.

Concilio Ecumenico Vaticano II, Costtuzione pastorale sulla Chiesa nel mondo contemporaneo Gaudium et spes in: Enchiridion Vaticanum. Documenti ufficiali del Concilio Vaticano II (1962-1965), vol. I, EDB Bologna 1981, pp. 772-965.

Concilio Ecumenico Vaticano II, Decreto su l'attivita missionaria della Chiesa Ad gentes in: Enchridion Vaticanum. Documenti ufficiali del Concilio Vaticano II (1962-1965), vol. I, EDB, Bologna 1981, pp. 608-695. 
Concilio Vaticano II, Dichiarazione sulla liberta religiosa Dignitatis humanae in: Enchiridion Vaticaanum. Documenti ufficiali del Concilio Vaticano II (1962-1965), EDB, Bologna 1981, pp. 578-605.

Congregazione per la Dottrina della Fede, Dichiarazione “Dominus Iesus", circa l'unicità e l'universalità salvifica di Gesù Cristo e della Chiesa, Libreria Editrice Vaticana, Città del Vaticano 2000.

Congregazione per la Dottrina della Fede, Dichiarazione "Dominus Iesus". Documenti e studi, Libreria Editrice Vaticana, Città del Vaticano 2002.

Congregazione per la Dottrina della Fede, Nota dottrinale su alcuni aspetti dell'evangelizzazione, Libreria Editrice Vaticana, Città del Vaticano 2007.

Fernandez F., In Ways Known to God, Vendrame Institute Publications, Shillong 1996.

Giovanni Paolo II, Esortazione apostolica post-sinodale circa Gesù Cristo, il Salvatore e la sua missione di amore e di servizio in Asia Ecclesia in Asia. Libreria Editrice Vaticana, Città del Vaticano 1999.

Pontificia Academia Theologica, "Gesù Cristo, via, verità e vita” (Gv 14,6). Per una rilettura della “Dominus Iesus”, PATH 1 (2002) n. 2, p. 141-370.

Schlier H., Lettera agli Efesini, Paideia, Brescia 1965. 